\title{
Performance Analysis of Wireless Network Control Systems Using Different Controllers
}

\author{
Ena'am F. Khuder, Sura N. Abd alrazaaq, Omar abd al-kareem \\ Computer Engineering Department \\ University of Mosul - IRAQ
}

\begin{abstract}
Recently Wireless communication technologies have strongly adopted in real-time industrial environments. The increasing availability of products and solutions based on the IEEE 802.11 standard make this kind of communication technology becomes more cost effective in distributed control systems. This paper describes real time control application over Wireless Local Area Network (WLAN) in Ad hoc mode using a Virtual Instrument VI environment (LabVIEW). Particularly the delay time in such networks is considered as a critical factor that has a significant effect on the system response time characteristics such as rise time $\left(\mathrm{T}_{\mathrm{r}}\right)$, settling time $\left(\mathrm{T}_{\mathrm{s}}\right)$. So in this paper we discussed the effect of using different controllers (such as P, PID, Fuzzy, ...) on the overall system performance. The experimental measurements show that the using of fuzzy controller gives best results where it can minimizes the effect of the network delay on the system behavior as much as possible.
\end{abstract}

Keywords: UDP/IP, WLAN, Remote real-time control, PID, Fuzzy.

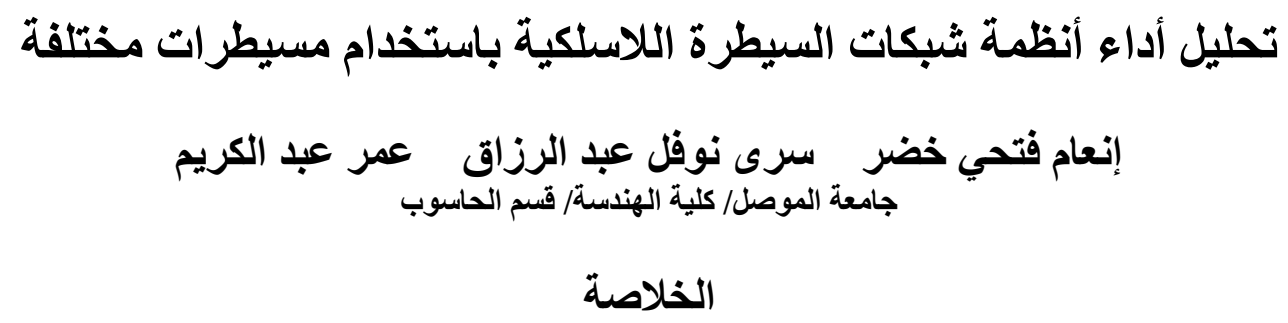

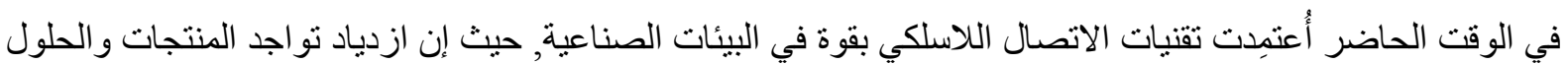

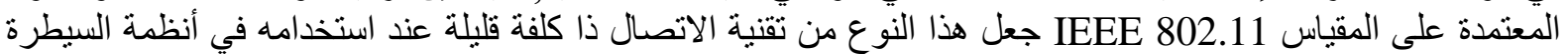

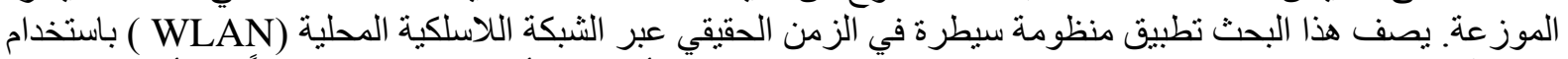

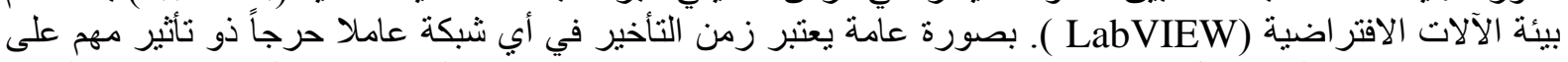

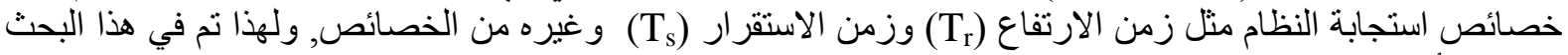

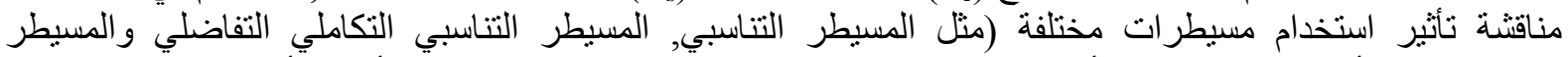

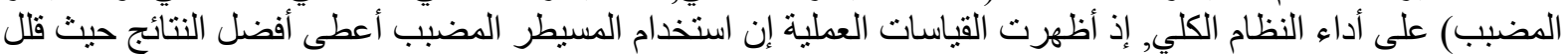
تأثير نأخبر الثبكة على تصرف النظماء النظام بأكبر قدر ممكن. 


\section{Introduction:}

There is currently an increasing interest in making wireless transmission technologies available not only in offices and homes, but also in industrial environments. Two especially attractive features are the reduced need for cabling and the potential for truly mobile stations. As the cost of advanced technology continues to drop, and various standards have been established, the future trend of wireless communications appears to be very promising [1].

One of the most popular wireless technologies used today is undoubtedly IEEE 802.11. The adoption of such a kind of networks, also known as wireless LANs (WLANs), is mainly suggested by some appealing features of theirs, such as, in particular, the high transmission speed, which provides more than an adequate bandwidth for many applications, and the close resemblance with conventional Ethernet. The last aspect means, that interoperability can obtain through simple and inexpensive access points (AP), which transparently interface wireless stations to wired segments [2].

The WLAN can provide the required high data rate links and flexible network topologies between the mobile industrial robots, automated machinery, and remote computer terminals in the factory, all without the cost of installing a wired data network. With the incentive of cost reduction of wireless devices, it is now a trend to develop the WLAN in factories to increase productivity and quality of goods [1][2].

In industrial environments, WLANs are used at present mainly to enable simple and costeffective maintenance and diagnostic functions. For instance, conventional APs are connected to the networks used at the factory shop floor in order to enable management devices to be temporarily connected to the system for reconfiguring the control software or changing the operating parameters [2].

WLAN was not originally designed for industrial applications and suffers from the same defections. Implementation of new standards such as 802.11a or 802.11g can rapidly enhance WLAN usability in real-time considerations - the new norms introduce faster transmission technologies. Another issue that might improve suitability of WLAN for industrial applications is design of a predictive controller dealing with varying delays or control algorithm that handles missing data or packet timeout[3].

For the sake of truth a number of wireless technologies have been developed recently for the use at the shop floor, that are aimed explicitly at connecting I/O modules to a PLC. InduraNET $\mathrm{p}$, and Wireless HART, Phoenix Contact's Wireless Interface RAD-ISM-900XD-BUS. The above solutions, which are not based on IEEE 802.11 technology, mostly rely on improved physical layers that make them more resistant to environmental noise and envisage some kind of scheduling scheme for improving determinism in network access. It is worth noticing that the bit rate in these networks is noticeably lower than in WLANs [4] [5].

The current work is investigating performance analysis of 802.11g WLANs standard in ad hoc mode in order to predict its behavior in control system. This paper examines the effect of wireless network delay on the stability of the control system (i.e. on rise time $\left(\mathrm{T}_{\mathrm{r}}\right)$, settling time $\left(\mathrm{T}_{\mathrm{s}}\right)$, ect). All the results compared with the results obtained in locally control.

This paper is organized as follows: Section II includes a literature review; Section III illustrates the WLAN standards. Section IV describes the structure of control system, section $\mathrm{V}$ includes the experimental measurements and simulation results with a discussion in section VI and finally section VII provides conclusions and future works. 


\section{Literature Review:}

The issues of stability analysis and networked-delays for networked control system (NCS) have received much concern for years. The network delay occurs when sensor, actuator and controllers exchange data over the network and it can be constant or time varying or random. For fast control loops, the delays drop the performance and can destabilize the system. So it is important to take the delays into account in the analysis and design of control system.

Azaam A. Mahammed, et. al. [6], the authors described a theoretical background and implementation of a real time position control scheme for a servo motor closed via an Ethernet network. They investigate the real time capability of Ethernet within the scope of the performance of UDP and TCP communication protocols.

Rusty $O$. Baldwin [7], the author developed Simulation models to study the performance of protocol RT-MAC (real time medium access control). RT-MAC is a novel, simple, and elegant MAC protocol for use in transmitting real-time data in point to point ad hoc WLAN. It is found that, in some cases, other factors such as the number of stations in the network and the offered load are more significant than the condition of the wireless channel.

Hong yi. et.al. [8], the author introduced a new protocol, prioritized carrier sense multiple access with collision avoidance, for real-time wireless local area networking. The paper focuses on scheduling the mixed traffic, especially time-critical feedback and control signals of the real-time control system.

P. Simacek et.al. [3], the authors described industrial real-time control application using wireless IP-based network. They focused on the ability of using WLAN standards in industrial application.

Leena Yiniemi [9], the author examined and compared the control performance of a laboratory-scaled process across three different wired networks and wireless ad-hoc network. The control experiments carried out by the laboratory-scaled flow process shows that the difference of the control performance between different networks is negligible.

\section{IEEE 802.11 WLAN Standard Overview:}

Since the beginning of wireless networking, many standards and technologies have been developed for WLANs. One of the most active standards organizations that address wireless networking is the Institute of Electrical and Electronics Engineers (IEEE).

In 1997, IEEE ratified the 802.11 standard, also known as Wireless Fidelity (Wi-Fi), for WLANs. The IEEE 802.11 standard supports three transmission methods, including radio transmission within the $2.4 \mathrm{GHz}$ band. In 1999, IEEE ratified two amendments to the 802.11 standard-802.11a and $802.11 \mathrm{~b}$ - that define radio transmission methods, and WLAN equipment based on IEEE 802.11 b quickly became the dominant wireless technology. IEEE $802.11 \mathrm{~b}$ equipment transmits in the $2.4 \mathrm{GHz}$ band, offering data rates of up to $11 \mathrm{Mbps}$. IEEE $802.11 \mathrm{~b}$ was intended to provide performance, throughput, and security features comparable to wired LANs. In 2003, IEEE released the 802.11g amendment, which specifies a radio transmission method that can use both the $2.4 \mathrm{GHz}$ and $5 \mathrm{GHz}$ bands and can support data rates of up to $54 \mathrm{Mbps}$. Additionally, IEEE 802.11g-compliant products are backward compatible with IEEE 802.11b-compliant products[10][11].

WLAN has two operation modes:

- Ad Hoc Mode: The ad hoc mode does not use APs. Ad hoc mode sometimes referred to as infrastructureless because only peer-to-peer stations are involved in the communications. 
- Infrastructure Mode: In infrastructure mode, an AP connects wireless stations to each other or to a distribution system, typically a wired network. Infrastructure mode is the most commonly used mode for WLANs.

\section{Structure of The Control System:}

Real time simulated model are used in order to represent a servomotor and a controller. Each of them is implemented in a separable computer and connected via wireless network. The basic scheme of our wireless control system (WCS) is shown in Figure (1) while the servo system represents a closed loop structure, so each computer sends and receives data in both directions at the same time. In this paper the server and the client term will be addressed on the controller and the model side respectively

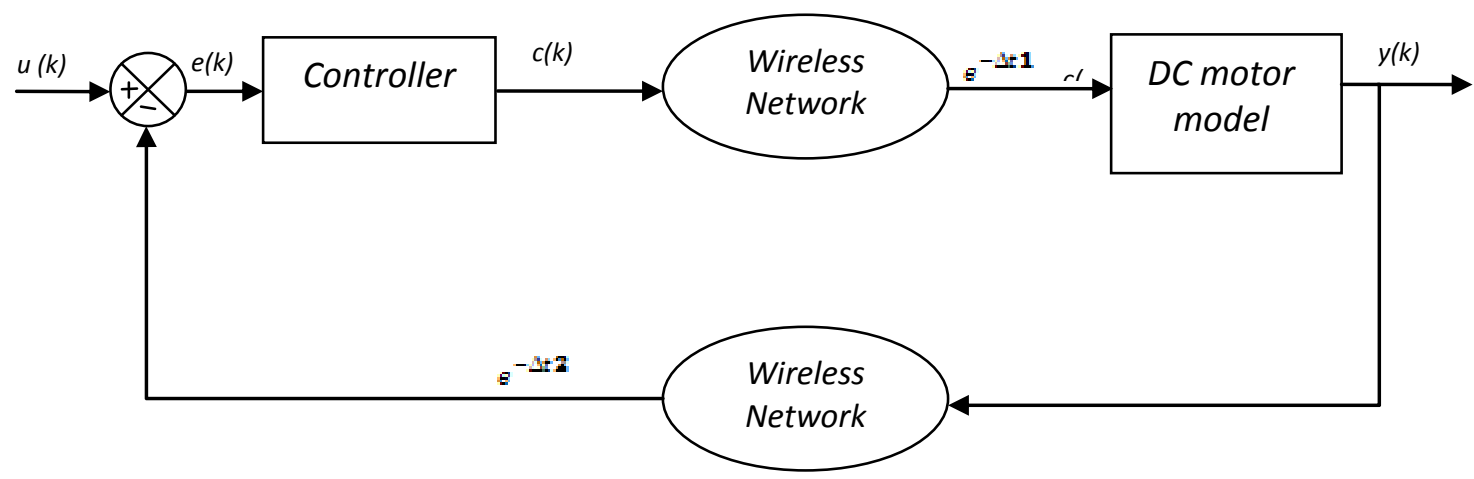

Figure (1): Block diagram of the closed loop WCS

The control application and communication program were developed using LabVIEW environment. The test sets consists of control parameters tests running on system using a remote computer via wireless network. The computer local to the process collects data on servo position from the servo motor to transmit it to the remote (client) computer via wireless network. The remote computer collects data on servo position, computes the response signal value and sends it back to the local computer, which transmits the control signal to the servo motor to actuate its response. The network delay $e^{-\Delta t_{1}}$ is produced through wireless network, for each sample has been transmitted from the controller to the motor, and $e^{-\Delta t z}$ represents the delay in the feedback path. As a result the total delay will influence the final time response characteristics and in this work we reduced it as much as possible by a predictive controller as will discussed in section VI.

\section{1- Software Description:}

The Lab VIEW software is used to develop the Virtual Instrument (VI) environment, which has two main parts, a front panel and a block diagram. The front panel is used for user interactions and display of results. The block diagram is the source code constructed using Lab VIEW's graphical programming language [13]. This language allowed a good solution to implement a wireless communication for industrial network, where the combination of virtual instruments and the network protocols can create more powerful; flexible and cost effectiveness automation systems. To implement the UDP/IP protocol there functions are connected with virtual wires and the data is transferred as a series of bytes in a string of characters [14]. Figure(3-a) shows the write UDP function that are used to instantiate a communication with a client computer at IP address 192.168 .1 .8 on port 61500 , the string < data> is written to this port and send to the client. 

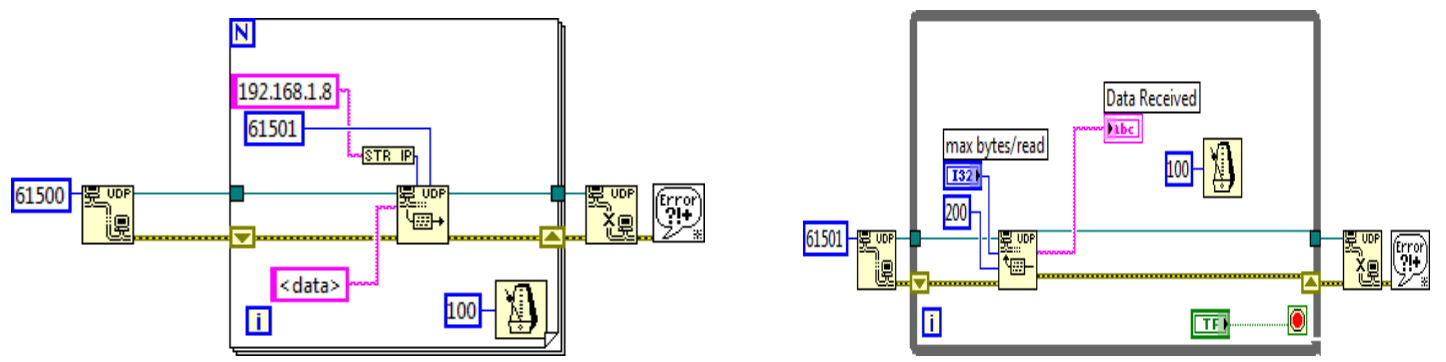

(a)

(b)

Figure (3): (a) UDP sender, (b) UDP receiver

The client or remote computer receives the data using UDP/IP read function; Figure ( 3-b) shows the UDP receiving section.

In theory, the datagrams can be sent of any size, however typically UDP would not be used to send large datagrams because it is not as reliable as TCP, so when all communications on a port are finished, the UDP Close function is used to free the system resources.

\section{2- Model Description:}

Direct-Current (DC) motors are one of the most widely used prime movers in the industry today and they are extensively used in control systems for many application. Therefore, DC motor is used as a controlled model in the designed (WCS). To establish the mathematical model for DC motors, Figure (2) shows the simplified block diagram for this model.

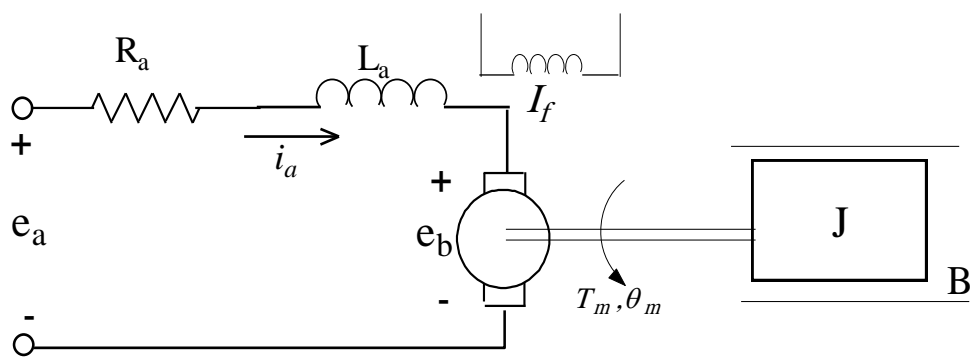

Figure (2): Simplified model for DC motor

The control signal that drives the motor represents the applied voltage $\left(\mathrm{e}_{\mathrm{a}}\right)$ on the armature circuit; the voltage source $e_{b}$ represents the back emf (electromotive force) in the armature when the rotor rotates by the angle $\theta_{m}$. The other parameters values of the system are given in the Table 1 [12], the open loop transfer function of the system is obtained by the following equation:

$G(s)=\frac{\theta_{m}(s)}{E_{a}(s)}=\frac{K_{s} A K_{i} n}{R_{a} B_{m e} s\left(1+\tau_{a} s\right)\left(1+\tau_{m e} s\right)+K_{b} K_{i} s}$

Where $\tau_{a}=\frac{L_{a}}{R_{a}}, \tau_{m e}=\frac{J_{m e}}{B_{m e}}$

$J_{m e}, B_{m e}$ are the equivalent inertia and viscous frictional coefficient seen by the motor respectively and 


$$
\begin{aligned}
& J_{m e}=J_{m}+n^{2} J_{l} \\
& B_{m e}=B_{m}+n^{2} B_{l} \\
& K_{b}=1.356 K_{i}
\end{aligned}
$$

Table 1: Parametric values of the model

\begin{tabular}{|l|l|l|}
\hline Parameters & Description & Value \\
\hline$K_{i}$ & torque constant & $0.5 \mathrm{Ib}-\mathrm{ft} / \mathrm{amp}$ \\
\hline$A$ & DC amplifier gain & 10 \\
\hline$R_{a}$ & armature resistance & $5 \Omega$ \\
\hline$L_{a}$ & armature inductance & $0.1 \mathrm{H}$ \\
\hline$J_{m}$ & motor shaft moment of inertia & $10^{-3} \mathrm{Ib}_{-} \mathrm{ft}-\mathrm{sec}^{2}$ \\
\hline$B_{m}$ & motor shaft friction & Negligible \\
\hline$B_{L}$ & load shaft friction & $0.1 \mathrm{Ib}-\mathrm{ft}-\mathrm{sec}^{2}$ \\
\hline$J_{L}$ & load moment of inertia & $0.1 \mathrm{Ib}-\mathrm{ft}-\mathrm{sec}^{2}$ \\
\hline$N$ & gear ratio & 10 \\
\hline$K_{s}$ & Sensitivity of error detector & $1 / 57.3$ volt / deg \\
\hline
\end{tabular}

\section{3-Controller Description}

There is no doubt that adding a controller to the system improves the performance of that system [16], but in this paper the influence of controller over wireless network are examined.

\section{- PID controller:}

First a PID controller is used in the server side to control the DC motor on the client side, where the setpoint is compared with the process variable which is represent the response of the motor that is obtained continuously at real time via wireless network by the designed VI that is shown in Figure(4). The PID controller calculates the controller action, according to the error signal and the PID parameters, then the resulting control signal $u(k)$ is transmitted again wirelessly to the client side and so on.

Figure(4) and Figure(5) show the block diagrams of the server and client VIs. this designed program is capable of executing PID controller as well as P or PI or PD controllers.

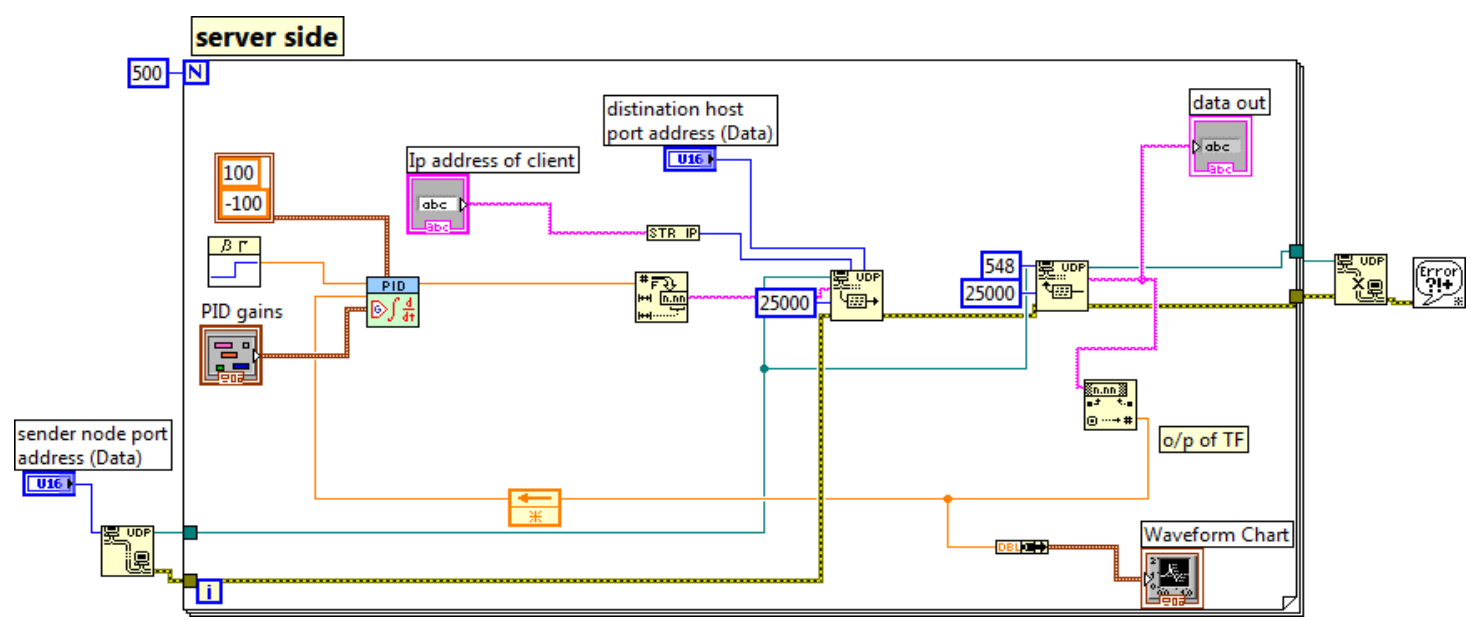

Figure (4): Block diagram of the controller VI at the server node 


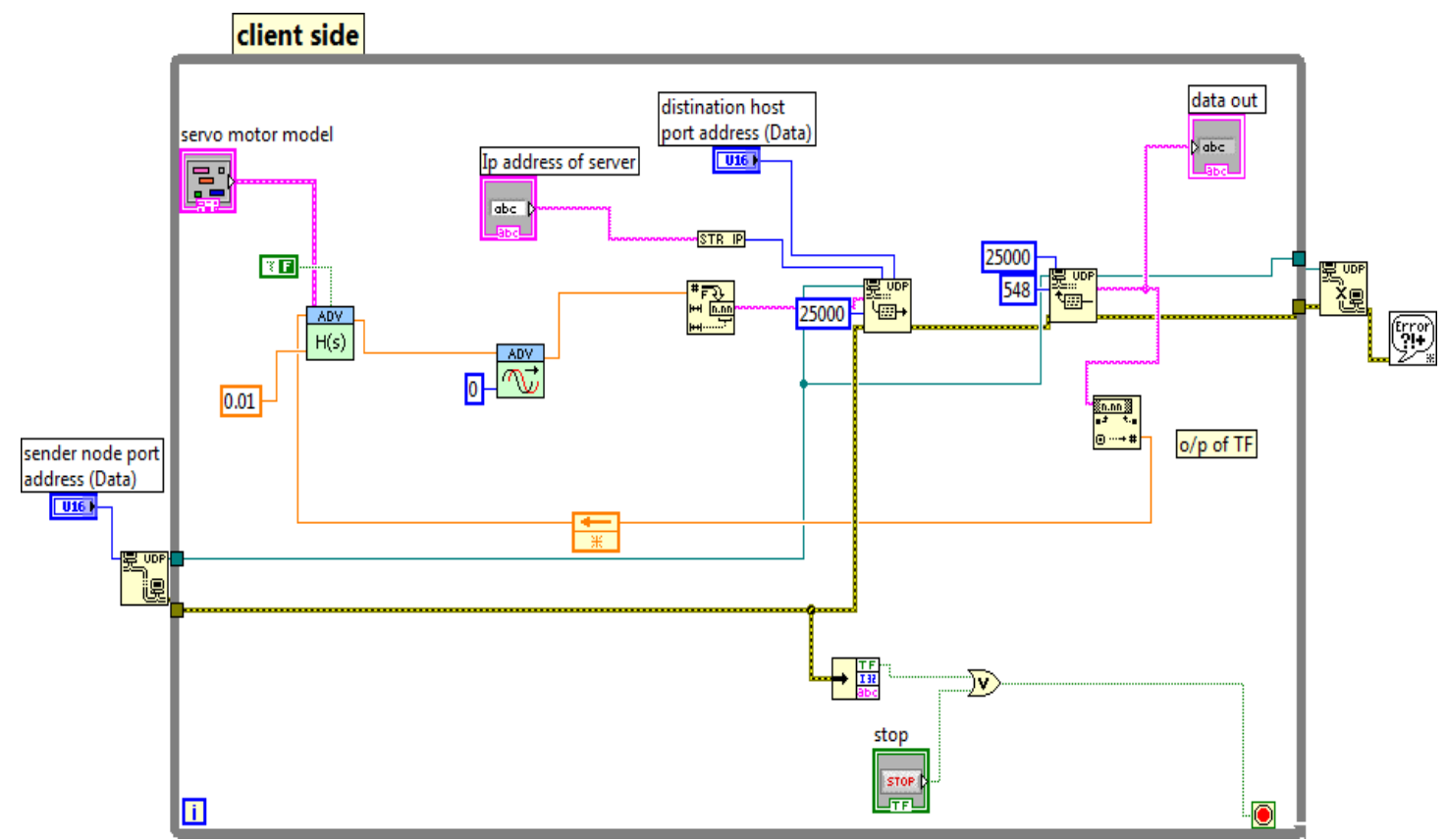

Figure (5). Block diagram of the controlled model on the client node

- Fuzzy controller:

A fuzzy logic controller (FLC) is used as a second technique to control the DC motor wirelessly to obtain better response. Any (FLC) comprises four principal components fuzzification, inference engine, knowledge base, and defuzzification [15].

In this paper the fuzzy controller have been designed with dual inputs and single output, the inputs are error between set point and the process variable $E(k)$, and change of error $\Delta E(k)$. The output of the fuzzy controller is the control signal $u(k)$ that is applied to the motor when it is sent via wireless network. This output can be expressed as a nonlinear function $(F)$ of the two inputs:

$\mathrm{U}(\mathrm{k})=\mathrm{F}(\mathrm{E}(\mathrm{k}), \Delta \mathrm{E}(\mathrm{k}))$

The (FLC) has been designed using a Fuzzy Logic Toolkit included in LabVIEW that enables us to integrate FLC into virtual instruments. This toolkit is utilized in this current work to design and implement rule-based fuzzy logic system for process control or expert decision making. Triangle membership functions (MFs) are used to subdivide the input and output universe and to define the degree of membership. As shown in Figure(6-a) and Figure( 6-b) there are seven states of the input position error $(E)$, position error's change $(\Delta E)$, and the output of the controller $(\mathrm{u})$.

The designed (FLC) is based on Mamdani fuzzy type, centre of gravity deffuzification method, Min - Max inference. The final step and the most important one is the creation of the fuzzy rules, where these rules are obtained from the experiences on the DC motor control and by depending on the human intuition, the FLC rules is shown in Table 2. 

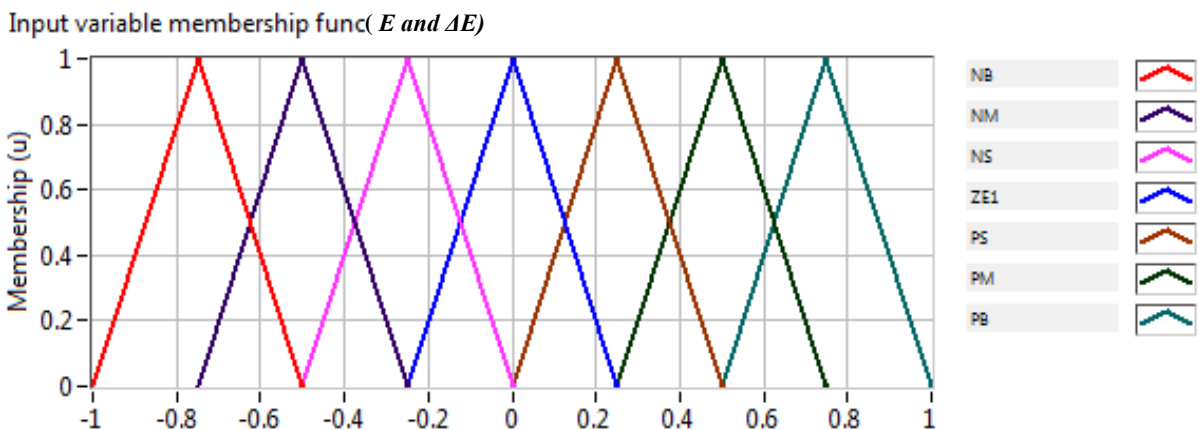

(a)
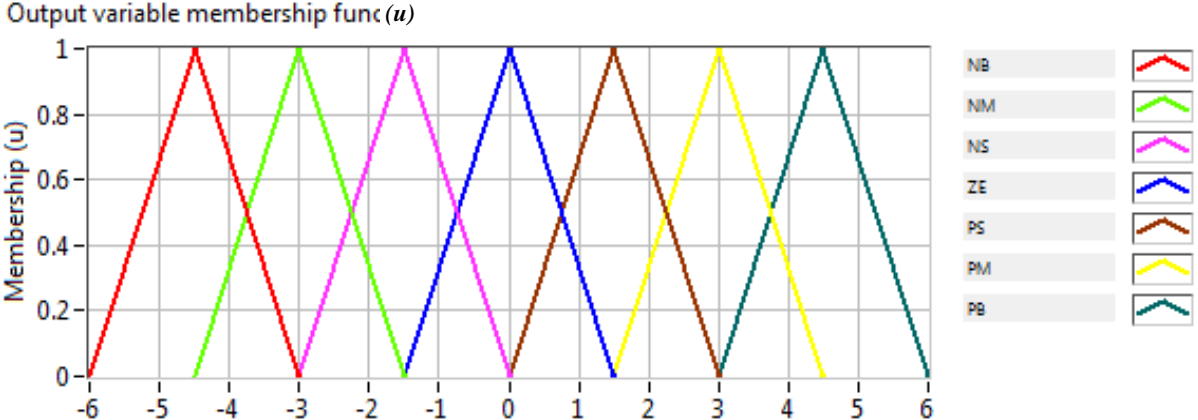

(b)

Figure (6): Inputs and output MFs of the designed (FLC)

Table 2: Fuzzy rules for the designed (FLC)

\begin{tabular}{|c|c|c|c|c|c|c|c|}
\hline$\Delta \mathbf{E}$ & $\mathrm{NB}$ & $\mathrm{NM}$ & $\mathrm{NS}$ & $\mathrm{ZO}$ & $\mathrm{PS}$ & $\mathrm{PM}$ & $\mathrm{PB}$ \\
\hline $\mathrm{NB}$ & $\mathrm{NB}$ & $\mathrm{NB}$ & $\mathrm{NB}$ & $\mathrm{NM}$ & $\mathrm{NS}$ & $\mathrm{NS}$ & $\mathrm{ZO}$ \\
\hline $\mathrm{NM}$ & $\mathrm{NB}$ & $\mathrm{NM}$ & $\mathrm{NM}$ & NM & NS & ZO & PS \\
\hline NS & NB & NM & NS & NS & ZO & PS & PM \\
\hline ZE & NB & NM & NS & ZO & PS & PM & PB \\
\hline PS & NM & NS & ZO & PS & PS & PM & PB \\
\hline PM & NS & ZO & PS & PM & PM & PM & PB \\
\hline PB & ZO & PS & PS & PM & PB & PB & PB \\
\hline
\end{tabular}

\section{Experimental Results:}

\section{Controllers Measurements :}

To implement the wireless control system, two computers (nodes) with LabVIEW program were located inside a same laboratory room about 25 square meters and connected peer to peer wirelessly. Wireless network is based on IEEE 802.11g WLAN standard with a maximum speed of $54 \mathrm{Mbps}$ and without any encryption. At the beginning, the possibility of the real time process control was tested without controller in ad-hoc mode. The output response is compared with the same one when the motor has been controlled locally, as shown in Figure (7). So to observe the effect of packets delay from server to client, and vice versa on the control system performance, we depended on measuring the response characteristics, rise time $\left(T_{r}\right)$ and settling time $\left(T_{s}\right)$, which, is the most affected parameters by the network delay, while the overshoot is slightly affected by this delay. In the second step, three controllers have been used individually to improve the DC motor response locally as well as to examine the effect of these controllers on the adhoc WCS. 


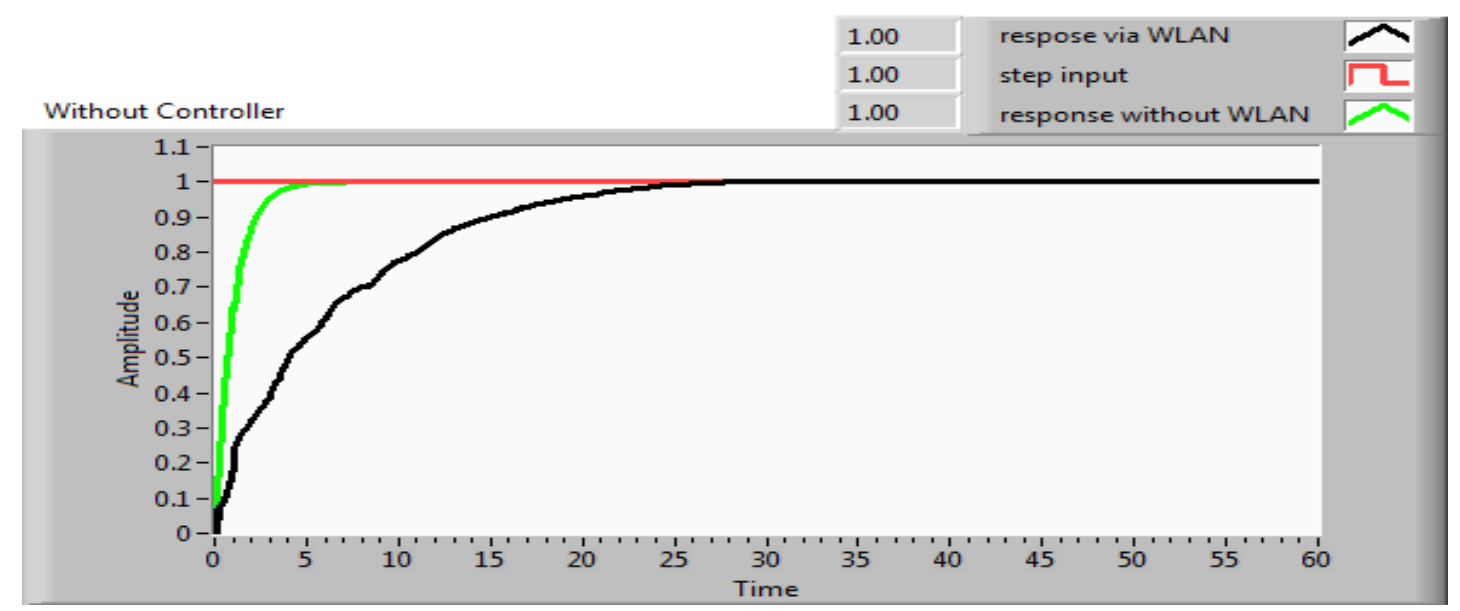

Figure 7: Effect of WLAN delay on DC motor response without controller

The results of the P and PID controllers are shown in Figure (8) and Figure (9) respectively, where the $K_{p}, T_{I}$ and $T_{D}$ parameters have been chosen based on Ziegler-Nicholas heuristic method [17]. In Figure(8-a), the fast mode of Ziegler-Nicholas is used with $\mathrm{Kp}=17.372$, as evident the response based wireless control has a very long settling time and when we increased the proportional gain slightly more, the system reached unstable state before the response based local control. Therefore to get little overshoot and to concentrate on the rise time and settling time in the response, the normal mode is depended instead as in Figure (8b).

In Figure (9) the system is controlled using PID controller. The response was improved compared with previous controller but the settling time is also considered long $\left(\mathrm{T}_{\mathrm{s}}=\right.$ $5.37 \mathrm{sec}$ ) in wireless based control. Figure (10) compares the DC motor response using fuzzy controller over wireless network and without network.

The comparison results of the response characteristics of the previous figures are summarized in Table 3 to show in details the differences in the system performance using different controllers.

Table 3: Response comparison results

\begin{tabular}{|l|l|l|l|l|}
\hline & \multicolumn{2}{|c|}{ Local control } & \multicolumn{2}{c|}{ Wireless control } \\
\hline & $\begin{array}{l}\text { Rise Time } \\
\mathrm{T}_{\mathrm{r}}(\mathrm{sec} .)\end{array}$ & $\begin{array}{l}\text { Settling Time } \\
\mathrm{T}_{\mathrm{s}}(\text { sec. })\end{array}$ & $\begin{array}{l}\text { Rise Time } \\
\mathrm{T}_{\mathrm{r}}(\mathrm{sec} .)\end{array}$ & $\begin{array}{l}\text { Settling Time } \\
\mathrm{T}_{\mathrm{s}}(\mathrm{sec} .)\end{array}$ \\
\hline without controller & 1.45 & 4.13 & 15.79 & 23.15 \\
\hline $\mathrm{P}$ controller $\mathrm{Kp}=17.372$ & 0.05 & 1.18 & 0.17 & 15.80 \\
\hline $\mathrm{P}$ controller $\mathrm{Kp}=4.516$ & 0.27 & 0.37 & 3.14 & 10.68 \\
\hline $\begin{array}{l}\text { PID controller } \\
\mathrm{K}_{\mathrm{p}}=6.254, \mathrm{~T}_{\mathrm{I}}=5.152, \mathrm{~T}_{\mathrm{d}}=0\end{array}$ & 0.16 & 0.56 & 0.77 & 5.37 \\
\hline Fuzzy controller & 0.15 & 0.39 & 0.15 & 0.40 \\
\hline
\end{tabular}




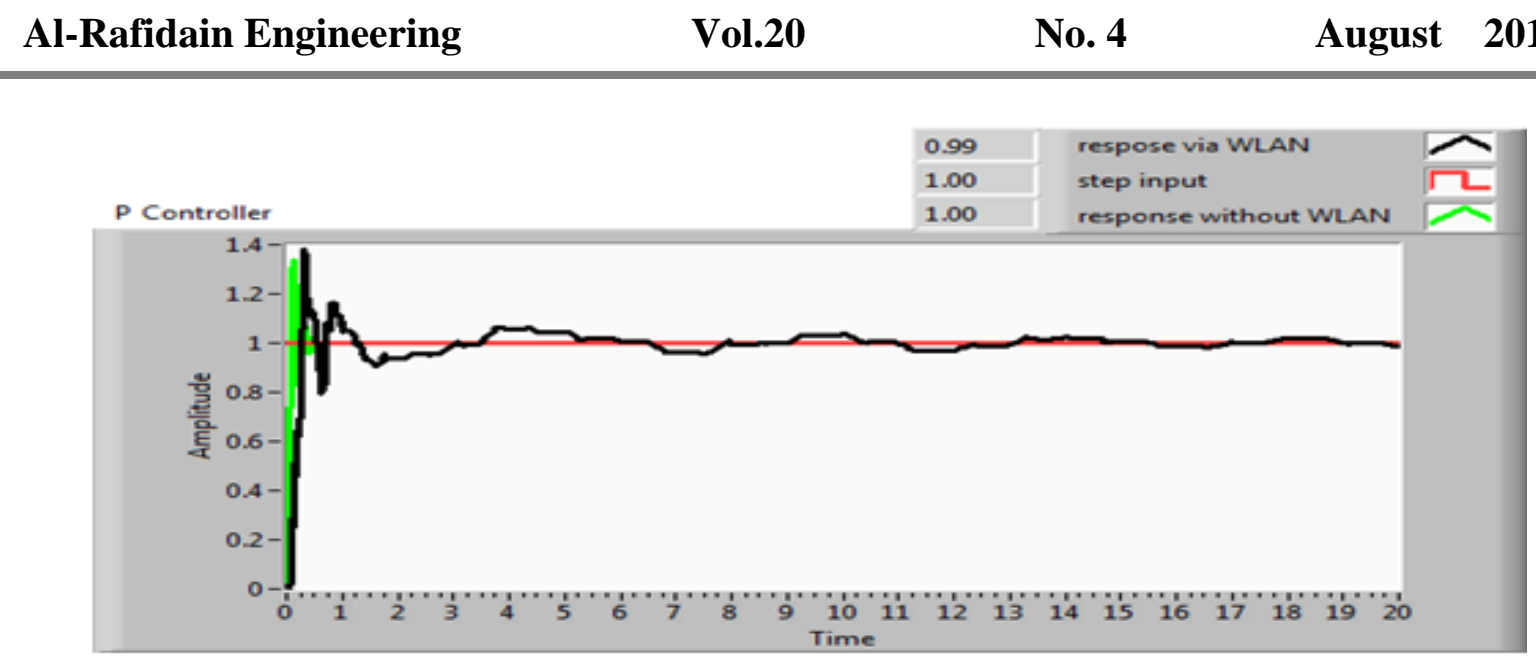

a- Fast mode

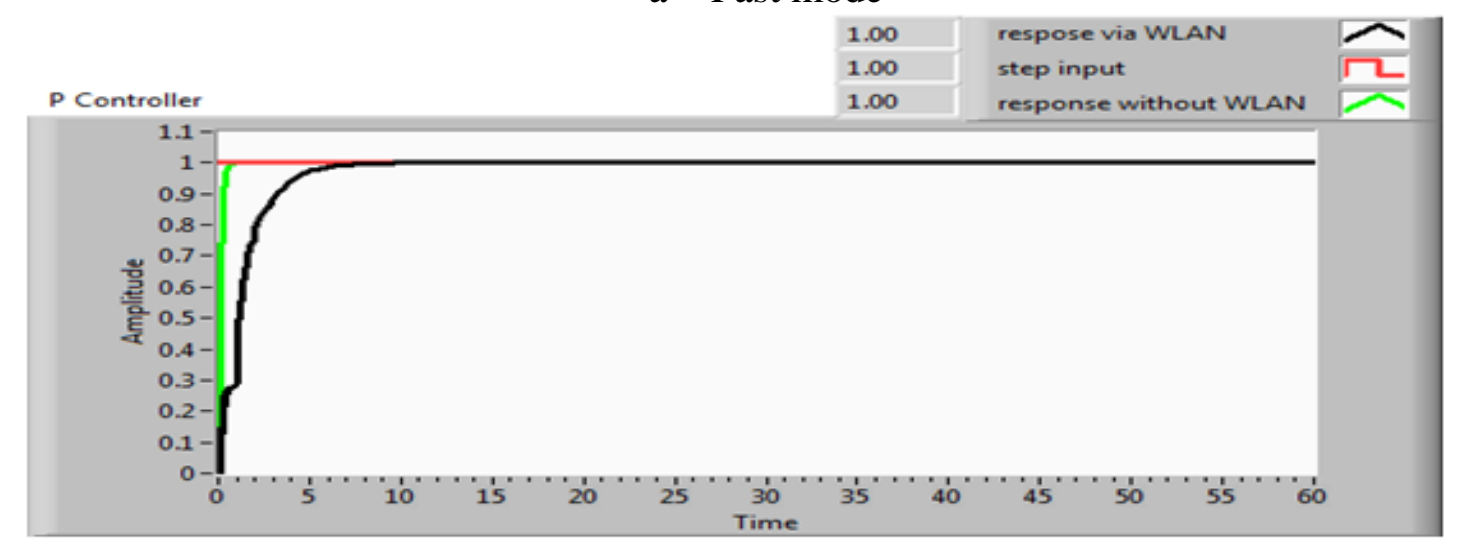

b- Normal mode

Figure 8. DC motor response comparison using $\mathrm{P}$ controller

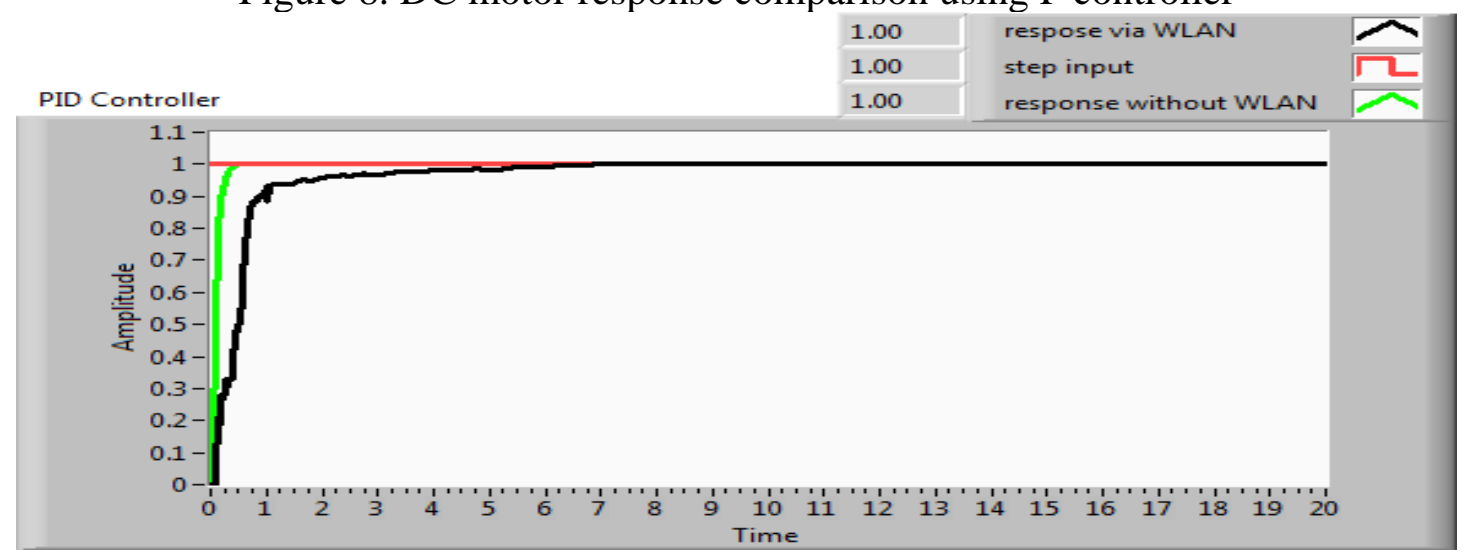

Figure 9. DC motor response comparison using PID controller

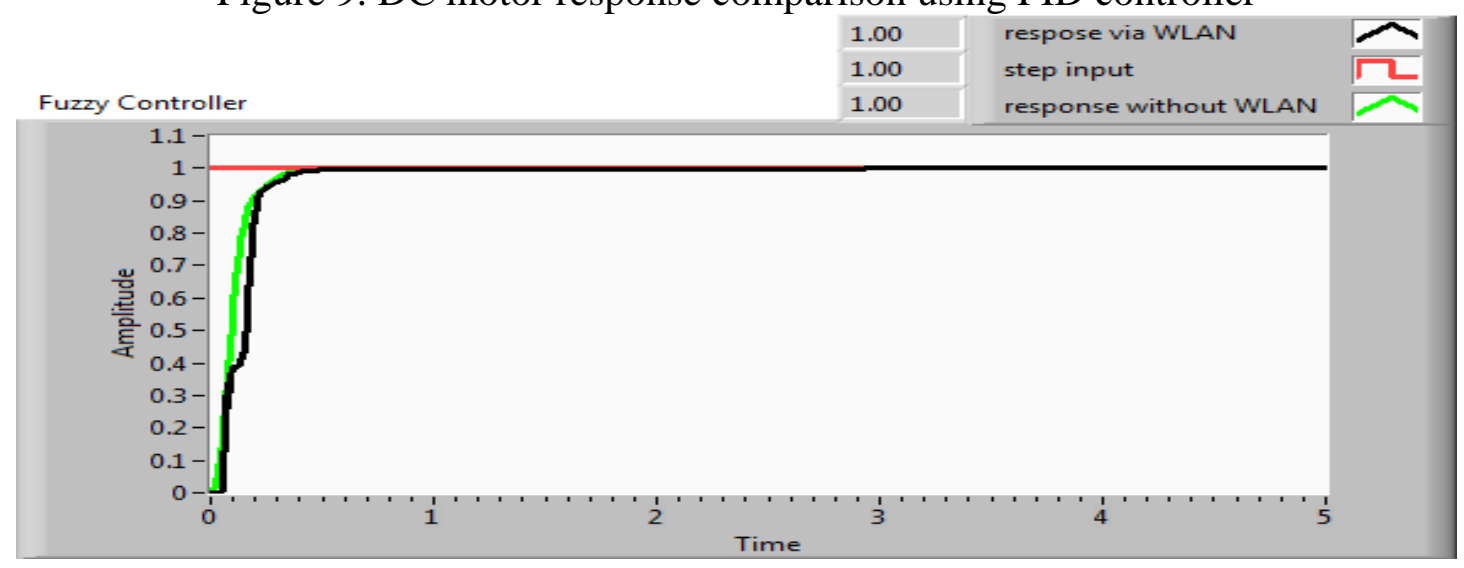

Figure (10): DC motor response comparison using fuzzy controller 


\section{Wireless Network Measurements:}

The Wire shark software is used to monitor the performance of WCS. A sample of this monitoring is shown in Figure (A-1). The two most important performance metrics are through put and network delay, through put is a rate at which bits are transformed from a source to a final destination. It is measured in bits per second, while the network delay represents the time between one sample and another (which is represent a time when the sample enter the network until it is used in the destination node includes the computational or the processing delay time of the program which can be embedded in the total delay $(\mathrm{T})$.

Under this assumption, we get two different delay times because the server node consumes long time, since the controller at this node need longer processing time compared with the client.

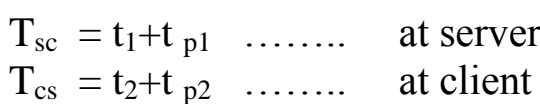

Where:

$\mathrm{T}_{\mathrm{sc}}$ : delay time from the server to the client.

$\mathrm{T}_{\mathrm{cs}}$ : delay time from the client to the server.

$\mathrm{t}_{1}, \mathrm{t}_{2}$ : network delay time from server to client and vice versa respectively.

$\mathrm{t}_{\mathrm{p} 1}, \mathrm{t}_{\mathrm{p} 2}$ : processing time at the server and the processing time at the client.

For one

sample

Figure (11) shows a shot of the samples delay times of $\mathrm{T}_{\mathrm{sc}}$ and $\mathrm{T}_{\mathrm{cs}}$, where the horizontal axis represents the frame number and the vertical axis represents the delay (T) in seconds. The average values of these times can be concluded where $T_{c s}$ and $T_{s c}$ is about $0.330 \mathrm{~ms}$ and $1.523 \mathrm{~ms}$ respectively.

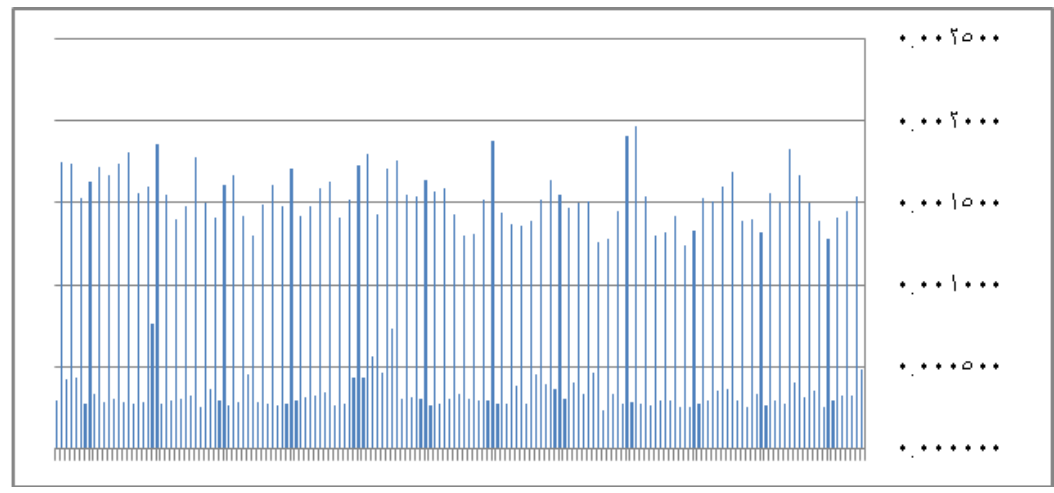

Figure (11): a shot of samples delay for WCS

For all tests, the packet size send is max 50 byte and the average bit per second (throughput) is about $0.146 \mathrm{M}$ bitlsec. also all packets extremely to be delivered successfully.

\section{Discussion and Analysis:}

In practice for the PID controller, whatever we changed the values of the PID parameters the difference in motor response between local control and wireless control was remained constant. This is because the PID controller depends on the error signal only to produce the control signal, but in the WCS; the error is decreased slowly. In other word the speed of the changing in error is important condition to decide the controlling signal. So the fuzzy 
controller is the best technique to achieve this criteria. Where this controller depends on the error, and the change of error as a antecedent in the controller rules to decide the output. Thus the firing rules will be different comparing with local control under same conditions, because the speed of change in error is different in WCS. Therefore, the fuzzy controller take the reaction depend on these two antecedents $(\mathrm{E}, \Delta \mathrm{E})$ and this is lead to change the controlling signal of the motor compared with local control, therefore the fuzzy controller is the best one in WCS.

According to measured results, following perception can be discussed: the delay time appears to be slightly random, this is according to usual disturbances in the building; however this small change in delay does not has a sensible effect on the performance of WCS. On the other hand it can be declared that the maximum delay time reached to $(1.957 \mathrm{~ms})$ (which includes the execution time of the program at server node) and this time delay is in the range of real-time delay constrains in industrial applications [18].

\section{Conclusions and Future Works:}

This paper involved the implementation of the closed loop wireless control system in real time environment using LabVIEW software, where the network delay induced in such a network and effected on the overall system response. From the experimental results it is evident that the time response characteristics $\left(T_{r}\right.$ and $\left.T_{s}\right)$ became longer when the DC motor was controlled wirelessly, but when the controller was used the system performance improved locally as well as wirelessly. The designed fuzzy controller was the best one that converts the wireless network induced delay system into a network. Free delay form by minimizing this delay indirectly (because it changes the control signal value) in such a way that it is a slightly different between local control and wireless control, with the same rise time 0.15 seconds and with 0.01 seconds different in settling time.

Current work brought many new ideas for future development of the project such as implementing a real-time model of a process as well as using different wireless standards dealing with varying delay.

\section{References:}

1. K.Sang , K.Fung , S. Kwong, "Wireless Communication Network Design in IC Factory", IEEE transactions on Industrial Electronics, vol. 48, no. 2, April 2001.

2. G. Cena, L. Seno, A. Valenzano," On the Performance of IEEE 802.11e Wireless Infrastructures for Soft-Real-Time Industrial Applications", IEEE transactions on industrial informatics, vol. 6, no. 3, August 2010.

3. Willig, M. Kubisch, Ch. Hoene, M. Hoene, A. Wolisz" Measurements of a Wireless Link in an Industrial Environment Using an IEEE 802.11-Compliant Physical Layer", IEEE transactions on industrial electronics, vol. 49, no. 6, December 2002.

4. P. Simacek, "Real-Time Control using Wireless Local Area Network", master thesis, Tampere University of Technology, Finland, 2002.

5. G. Cena, I. Bertolotti,"Evaluation of Response Times in Industrial WLANs", IEEE transactions on industrial informatics, vol. 3, no. 3, August 2007.

6. A. Mohammed, A. Mukhlif, "TCP vs. UDP protocols Ethernet based Servo Motor Control on LabVIEW Environment", 1'st International Engineering Science Conference IESC'08,2 November 2008. 
7. R. O. Baldwin" Improving the Real-Time Performance of a Wireless Local Area Network", Doctor of Philosophy in Electrical Engineering, Blacksburg, Virginia, June 23, 1999.

8. H. Ye, G. C. Walsh ," Real Time Mixed Traffic Wireless network", IEEE transactions on industrial electronics, vol. 48, no. 5, October 2001.

9. L. Yliniemi , "Process Control via Network", Proceedings of the 17th World Congress The International Federation of Automatic Control Seoul, Korea, July 6-11, 2008.

10. B. Forouzan," Data communications and networking", McGraw-Hill, 2007.

11. M. Gast,"802.11 Wireless networks", O’REILLY. ISBN: 0-596-00183-5.

12. Kuo Benjamin C. and Farid Golnaraghi "Automatic Control Systems" prentice-Hall. Inc., $3^{\text {rd }}$ edition, 1975.

13. Bitter R., Mohiuddin T. and Nawrocki M. , "LabVIEW Advanced Programming Techinques", CRC Press LLC, 2001.

14. National Instruments, Notes on UDP Communication in LabVIEW, Application Note on NI Developer Zone [online].Available on: http://zone.ni.com/devzone/cda/tut/p/id/4950.

15. Cirstea M. N. , Dinu A. , Khor J.G. , McCormick M. " Neural and Fuzzy Logic Control of Drives and Power Systems", Newnes , ISBN 0750655585 , 2002.

16. Sura N. A. "Design and Implementation of a Fuzzy Controller with Self Tuning factors using LabVIEW" Master thesis, University of Mosul, Computer Engineering Dept., 2008.

17. National Instruments Corporation "LabVIEW ${ }^{\mathrm{TM}}$, PID and Fuzzy Logic Toolkit User Manual”, 372192D-0, June 2009.

18. G. Cena, L. Seno, A. Valenzano, C. Zunino, "On the Performance of IEEE 802.11e Wireless Infrastructures for Soft-Real-Time Industrial Applications", IEEE Transactions on Industrial Informatics, vol. 6, no. 3, August 2010.

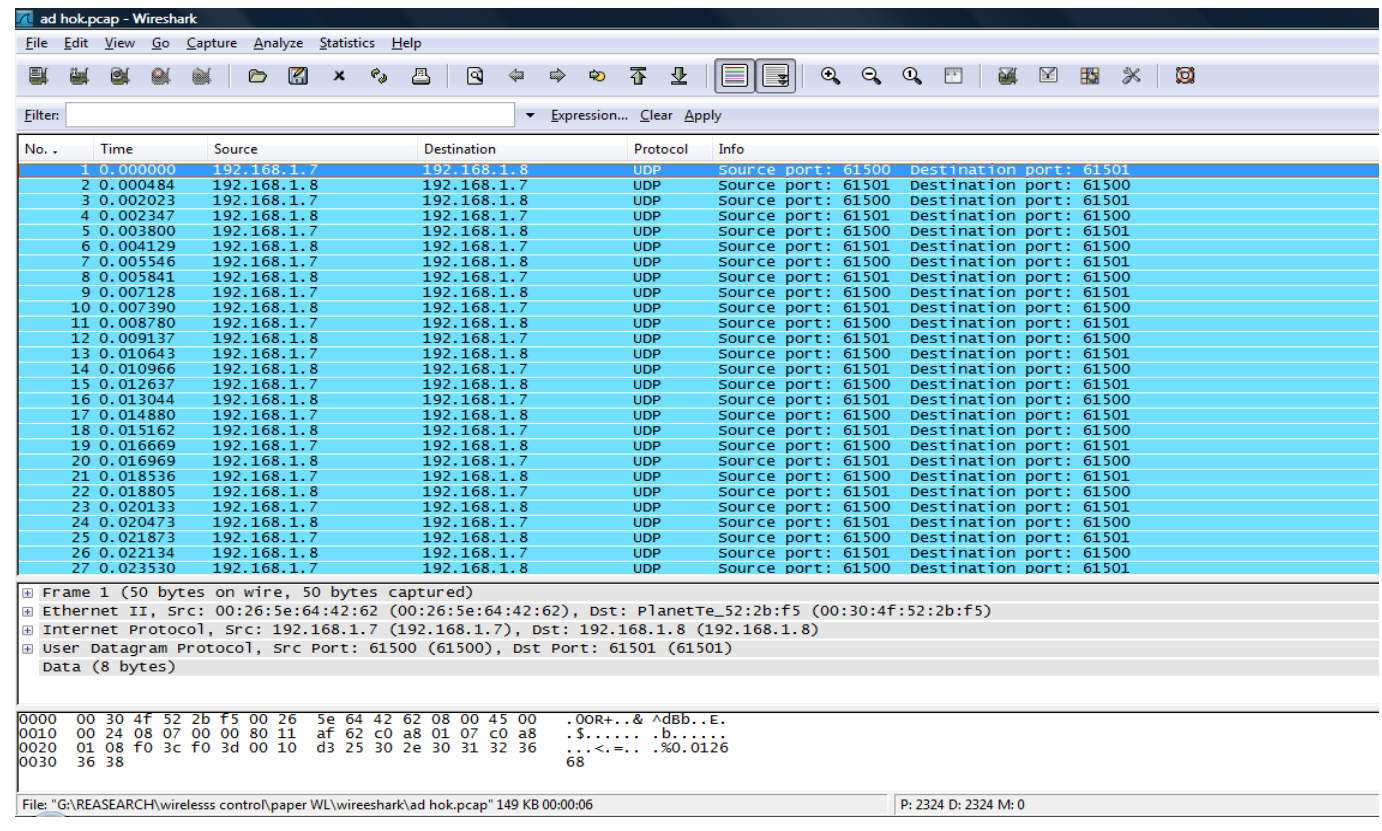

Figure (A-1) a snapshot of the monitoring window for WCS

The work was carried out at the college of Engineering. University of Mosul 